\title{
Eye-on-Hand Calibration Method for Cable-Driven Parallel Robots
}

\author{
Nicolas Tremblay ${ }^{1}$, Kaveh $\mathrm{Kamali}^{2}$, Philippe Cardou ${ }^{1}$, Christian Desrosiers ${ }^{2}$, \\ Marc Gouttefarde ${ }^{3}$, and Martin J.-D. Otis ${ }^{4}$ \\ 1 Laboratoire de Robotique, Département de génie mécanique, Université Laval, \\ Québec City, QC, Canada \\ nicolas.tremblay.31@ulaval.ca, pcardou@gmc.ulaval.ca \\ 2 École de technologie supérieure, Montréal, QC, Canada \\ kaveh.kamali.1@ens.etsmtl.ca, christian.desrosiers@etsmtl.ca \\ ${ }^{3}$ LIRMM, University of Montpellier, CNRS, Montpellier, France \\ marc.gouttefarde@lirmm.fr \\ 4 Laboratoire d'automatique et d'interaction 3D multimodale intelligente (LAIMI), \\ Département des Sciences Appliquées, Université du Québec à Chicoutimi (UQAC), \\ Chicoutimi, QC, Canada \\ martin_otis@uqac.ca
}

\begin{abstract}
Estimating the geometric parameters of a cable-driven parallel robot (CDPR) can be a labour intensive process or one that requires expensive sensors. This paper presents a low-cost method for estimating initial cable lengths and fixed cable attachment points of CDPRs. The proposed approach relies on the detection, mapping and localisation of fiduciary markers in the robot environment using a camera attached to the end effector. This paper additionally tackles the generation of a list of reachable calibration poses and presents a control scheme allowing the CDPR to reach those. Experiments are also carried out to assess the performance of the proposed calibration method. It appears that the proposed eye-on-hand method is more accurate than a previously reported method relying solely on cable-length measurements.
\end{abstract}

Keywords: Cable-driven parallel robot, parameter identification, experimental testing, vision-based calibration

\section{Introduction}

Cable-driven parallel robots (CDPRs) are a type of parallel robots where rigid links are replaced by cables, known for their advantageous payload to weight ratio which allows for higher dynamic capabilities, potentially larger translational workspace, portability and reconfigurability. In order to fully benefit from these capabilities, the CDPR geometric parameters have to be determined accurately, which can prove to be difficult on a medium to large scale. Indeed, a poor knowledge of these parameters inevitably degrades the positioning accuracy of the end effector. 
Excluding conceptually simple but impractical direct measurement of the geometry, some techniques have been developed for the Gough-Stewart platform calibration, but also apply to CDPRs. A popular and straightforward method uses measurements of the end effector pose in order to minimise inverse kinematic residuals [16]. The required measurements are often obtained using external sensors, such as a redundant leg [12], a theodolite [15] or a camera [13]. The calibration can also be performed by imposing additional kinematics constraints to render the internal sensors redundant [5].

Methods were proposed specifically for calibrating fully-constrained CDPRs, without the need for external sensors $[1,2,6]$. The use of force sensors, allowing for simultaneous stiffness identification, is also addressed in [10]. A few different calibration algorithms are also tested experimentally in [1]. However, because they rely on measurement redundancy, these methods algorithms cannot be applied to cable-suspended CDPRs. Without proper initial estimation of the solution to the geometric identification problem, the calculation time for a large number of calibration poses can also be considerable and the accuracy of the solution cannot be guaranteed.

An alternative to these self-calibration methods consists in resorting to external sensors to measure directly the end effector pose. Over the large workspace of CDPRs, one could use traditional surveying sensors such as theodolites or total stations but their use is time-consuming. This problem can be resolved by resorting to automated measurement devices such as laser trackers [4] or motion capture systems, which can be used to perform different measurements either to directly measure the geometry or to establish the robot posture. However, while these solutions currently seem to prevail within academia, the cost of such systems may be of the same order of magnitude as that of the CDPR itself. Hence, there is a true incentive to find an inexpensive methodology for the calibration of CDPRs.

It is well known that a calibrated camera can be used to estimate the position and orientation of a size-known object expressed in its own reference frame and vice versa. Eye-on-hand, or eye-in-hand calibration is performed using a moving camera, often attached to the robot end effector and a fixed target. This method is used in particular to calibrate serial robots [7]. In the case of a CDPR, one advantage of this method is that only one camera is needed to perform a calibration over a large workspace. Moreover, the placement of the camera is simple and space efficient, considering that the camera on the end effector can move across the whole workspace. This avoids having to position one or more fixed cameras in such a way that it is possible to accurately track targets fixed on the end effector. Considering those potential benefits, the present paper outlines a new approach to CDPR calibration using this technique.

In section 2, the calibration process and the generation of a list of poses used to gather data is addressed. Then, in section 3, a control scheme for reaching the calibration poses is described. Finally, experiments are presented and the results are discussed in section 4 . 


\section{Calibration}

The goal of the calibration process is to identify the geometry of a given CDPR. In the current case, we work under the hypothesis of massless and inextensible cables. Fig. 1 shows the general geometric model of a CDPR driven by $m$ cables. We assume that the cable lengths are measured using relative encoders, so that the total length of the $i^{\text {th }}$ cable is expressed as $\rho_{i}=\Delta \rho_{i}+\rho_{i, 0}$, where $\rho_{i, 0}$ is the cable length when the robot controller is first powered on after a reconfiguration. The geometric loop-closure equation, for a given pose $X=\{\mathbf{p}, \mathbf{Q}\}$ takes the form:

$$
\left(\Delta \rho_{i}+\rho_{i, 0}\right)^{2}=\left(\mathbf{p}+\mathbf{Q b}_{i}-\mathbf{a}_{i}\right)^{T}\left(\mathbf{p}+\mathbf{Q b}_{i}-\mathbf{a}_{i}\right),
$$

where $\mathbf{p}$ and $\mathbf{Q}$ are respectively the position vector and orientation matrix of the end effector. In the case of a CDPR, the unknown parameters are usually the position of the cable output points and the initial cable lengths, namely $\mathbf{a}_{i}$ and $\boldsymbol{\rho}_{i_{0}}$. A full calibration would include an identification of the end effector attachment points $\mathbf{b}_{i}$ but since the latter is generally small, we consider that these parameters can be established beforehand using conventional measuring techniques. Regardless of the value of $m$, if a spatial mechanism is considered, the constraint $n \geq 4$ must be satisfied in order to avoid underdetermination of the system, where $n$ is the number of calibration poses.

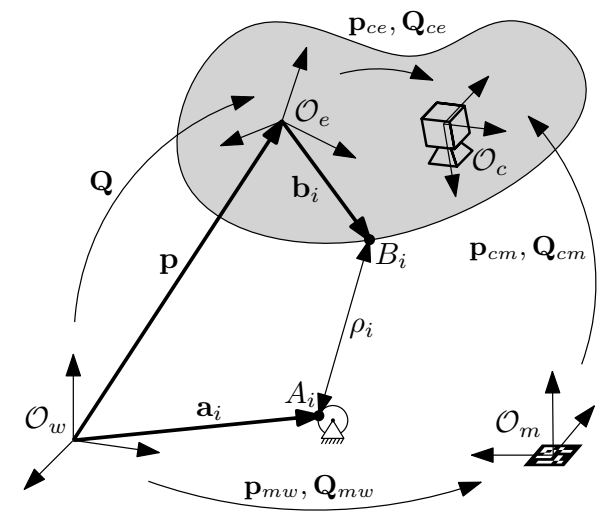

Fig. 1. Cable robot geometric model for eye-on-hand calibration

The residual of the loop-closure equation, for each measurement $j$, is written

$$
u_{i, j}=\left\|\mathbf{p}_{j}+\mathbf{Q}_{j} \mathbf{b}_{i}-\mathbf{a}_{i}\right\|_{2}^{2}-\left(\Delta \rho_{i, j}+\rho_{i, 0}\right)^{2} .
$$

where $\Delta \rho_{i, j}$ is directly obtained from the relative encoders and $\mathbf{p}_{j}$ and $\mathbf{Q}_{j}$ are provided by an external sensor. The calibration problem is then written as a nonlinear least squares optimisation, namely

$$
\underset{\mathbf{a}_{i}, \rho_{i, 0}}{\operatorname{minimise}} \sum_{i=1}^{m} \sum_{j=1}^{n} u_{i, j}^{2}
$$


when considering all cables $i=1, \ldots, m$. The next section provides details concerning the external measurements for every calibration pose.

\section{$2.1 \quad$ Eye-on-Hand Calibration}

In the work reported in this paper, this external sensor is an embedded camera, which must provide enough data to estimate the end effector pose $\left(\mathbf{p}_{j}, \mathbf{Q}_{j}\right)$. The detection of fiduciary markers is the chosen approach because it is simple and it allows for fast processing and reliable positioning.

As the end effector is intended to cover a large workspace, the use of a single marker would result in poor positioning accuracy and would be limited to only a small part of the workspace. This problem could be solved by using an array of different markers of known relative poses but the manufacturing of such objects would be impractical or prone to errors. Instead, we choose to spread uniquely identified markers throughout the robot workspace, without prior knowledge of their relative pose. The existing ArUco C++ library [3,14] can be used for marker generation and detection and the corresponding marker mapper [11] tool can easily be adapted, which makes it well suited for the current application. This library allows for localisation and mapping of the markers. The resulting map can then be used to estimate the camera pose. In such a case, the markers can be removed after the calibration is completed.

The end effector pose can be obtained from the measured camera pose through the rigid transformations $\mathbf{p}_{c e}$ and $\mathbf{Q}_{c e}$, namely,

$$
\begin{aligned}
\mathbf{p} & =\mathbf{p}_{m w}+\mathbf{Q}_{m w} \mathbf{p}_{c m}+\mathbf{Q}_{m w} \mathbf{Q}_{c m} \mathbf{p}_{c e} \\
\mathbf{Q} & =\mathbf{Q}_{m w} \mathbf{Q}_{c m} \mathbf{Q}_{c e}^{T}
\end{aligned}
$$

where $\mathbf{p}_{c m}$ and $\mathbf{Q}_{c m}$ are obtained using the camera pose reconstructed by the marker mapper. If $\mathbf{p}_{m w}$ and $\mathbf{Q}_{m w}$ are not known beforehand, it is possible to choose $\mathcal{O}_{w}$ such as it is coincident with $\mathcal{O}_{m}$, which leads to $\mathbf{p}_{m w}=\mathbf{0}$ and $\mathbf{Q}_{m w}=\mathbf{1}_{3 \times 3}$, thus eliminating any possibility of measurement errors between the two reference frames.

The constant values $\mathbf{p}_{c e}$ and $\mathbf{Q}_{c e}$ must be determined prior to calibration. These can be obtained by observing a marker with the end effector camera and comparing the resulting $\mathbf{p}_{c m}$ and $\mathbf{Q}_{c m}$ measurements to the measured position and orientation of the end effector with respect to the marker reference frame. For fully-constrained CDPRs, this transformation can also be estimated alongside the calibration by simply adding the parameters $\mathbf{p}_{c e}$ and $\mathbf{Q}_{c e}$ to the decision variables of the optimisation problem of Eq. 3 .

\subsection{Generation of a List of Reachable Calibration Poses}

In order to perform an autonomous calibration, it is essential to generate a pool of poses $X_{j}=\left\{\mathbf{p}_{j}, \mathbf{Q}_{j}\right\}$, which can be reached from an initial pose $X_{0}=\left\{\mathbf{p}_{0}, \mathbf{Q}_{0}\right\}$, located inside the workspace. To be feasible, the trajectory must be included within the wrench-feasible workspace and free of collision between cables. The 
proposed method works by projecting $X_{r}$, a pose randomly generated inside a 6-dof box, towards $X_{0}$, on the workspace of the robot.

However, in order to find reachable poses, an initial estimation of the parameters to calibrate is needed. In the context of this paper, the latter is obtained using a simple measuring tape, but other viable and affordable solutions are available, such as ultra-wideband positioning systems and inclinometers.

Let us define the trajectory between $X_{0}$ and $X_{r}$ as a straight-line motion of point $\mathbf{p}_{0}$ combined with a constant angular velocity of the end effector. Such a motion is parameterised as

$$
\begin{aligned}
\mathbf{p}(\lambda) & =\mathbf{p}_{0}+\lambda\left(\mathbf{p}_{r}-\mathbf{p}_{0}\right) \\
\mathbf{Q}(\lambda) & =\mathbf{Q}_{t}(\lambda) \mathbf{Q}_{0}
\end{aligned}
$$

where $0 \leq \lambda \leq 1$ and

$$
\mathbf{Q}_{t}(\lambda)=\mathbf{e e}^{T}+\cos (\lambda \phi)\left(\mathbf{1}_{3 \times 3}-\mathbf{e e}^{T}\right)+\sin \lambda \phi \mathbf{E}
$$

is the rotation undergone by the end effector along its trajectory from its attitude at $\lambda=0$ to that at $\lambda$. We use

$$
\mathbf{E}=\operatorname{cpm}(\mathbf{e}), \quad \text { where } \quad \operatorname{cpm}(\mathbf{x}) \equiv \frac{\partial(\mathbf{x} \times \mathbf{y})}{\partial \mathbf{y}}
$$

so that $\mathbf{e}$ and $\phi$ respectively represent the rotation axis and the rotation angle. Upon solving Eq. (5b) for $\mathbf{Q}_{t}(\lambda)$ and setting $\lambda=1$, we obtain

$$
\mathbf{Q}_{t}(1)=\mathbf{Q}_{r} \mathbf{Q}_{0}^{T}
$$

Those axis and angle values can be then found first by defining

$$
\operatorname{vect}\left(\mathbf{Q}_{t}(1)\right) \equiv \frac{1}{2}\left[\begin{array}{l}
q_{t_{32}}-q_{t_{23}} \\
q_{t_{13}}-q_{t_{31}} \\
q_{t_{21}}-q_{t_{12}}
\end{array}\right] \quad \text { and } \quad \operatorname{tr}\left(\mathbf{Q}_{t}(1)\right) \equiv q_{t_{11}}+q_{t_{22}}+q_{t_{33}}
$$

and then using the properties

$$
\begin{aligned}
\operatorname{tr}\left(\mathbf{Q}_{t}(1)\right) & =1+2 \cos \phi, \\
\operatorname{vect}\left(\mathbf{Q}_{t}(1)\right) & =\sin \phi \mathbf{e} .
\end{aligned}
$$

For the purpose of satisfying the wrench-feasible criterion, we aim to find the maximum $\lambda=\lambda_{s}$ value that can be reached without any constraint violation. This can be ensured first by defining the cable direction for each cable

$$
\mathbf{c}_{i}\left(\lambda_{s}\right)=\frac{\mathbf{a}_{i}-\mathbf{p}\left(\lambda_{s}\right)-\mathbf{Q}\left(\lambda_{s}\right) \mathbf{b}_{i}}{\left\|\mathbf{a}_{i}-\mathbf{p}\left(\lambda_{s}\right)-\mathbf{Q}\left(\lambda_{s}\right) \mathbf{b}_{i}\right\|}
$$

and constructing the end effector pose-dependant wrench matrix

$$
\mathbf{W}\left(\lambda_{s}\right)=\left[\begin{array}{ccc}
\mathbf{c}_{1}\left(\lambda_{s}\right) & \cdots & \mathbf{c}_{m}\left(\lambda_{s}\right) \\
\mathbf{Q}\left(\lambda_{s}\right) \mathbf{b}_{1} \times \mathbf{c}_{1}\left(\lambda_{s}\right) & \cdots & \mathbf{Q}\left(\lambda_{s}\right) \mathbf{b}_{m} \times \mathbf{c}_{m}\left(\lambda_{s}\right)
\end{array}\right] .
$$


By imposing bounds over the cable tensions in the cables $t_{\min } \leq t_{i} \leq t_{\max }$ and the static equilibrium constraints, we then formulate the problem as follows

$$
\begin{aligned}
\text { maximise } & \lambda_{s} \\
\text { subject to } & \mathbf{t}_{\min } \leq \mathbf{t} \leq \mathbf{t}_{\max }, \\
& \mathbf{W}\left(\lambda_{s}\right) \mathbf{t}+\mathbf{w}_{e}=\mathbf{0}, \\
& 0 \leq \lambda_{s} \leq 1
\end{aligned}
$$

where $\mathbf{t}$ is the array of cable tensions and $\mathbf{w}_{e}$ is the wrench applied on the end effector by its environment.

A similar approach is used for considering the cable-cable interferences. Let $\zeta\left(\mathbf{p}_{k 1}, \mathbf{p}_{k 2}, \mathbf{p}_{l 1}, \mathbf{p}_{l 2}\right)$ be a function computing the distance between two line segments defined by their four end-points $\mathbf{p}_{k 1}, \mathbf{p}_{k 2}, \mathbf{p}_{l 1}$ and $\mathbf{p}_{l 2}$, as presented in [9]. The cables $k$ and $l$ are therefore represented by the line segments $\mathbf{p}_{k 2}-\mathbf{p}_{k 1}$ and $\mathbf{p}_{l 2}-\mathbf{p}_{l 1}$. These points can then be rewritten as functions of the geometry and of the trajectories described in Eqs. (5a) and (5b):

$$
\begin{aligned}
\mathbf{p}_{k 1} & =\mathbf{a}_{k}, \\
\mathbf{p}_{k 2} & =\mathbf{p}\left(\lambda_{k, l}\right)+\mathbf{Q}\left(\lambda_{k, l}\right) \mathbf{b}_{k}, \\
\mathbf{p}_{l 1} & =\mathbf{a}_{l}, \\
\mathbf{p}_{l 2} & =\mathbf{p}\left(\lambda_{k, l}\right)+\mathbf{Q}\left(\lambda_{k, l}\right) \mathbf{b}_{l} .
\end{aligned}
$$

A collision between two real cables occurs when $\zeta\left(\mathbf{p}_{k 1}, \mathbf{p}_{k 2}, \mathbf{p}_{l 1}, \mathbf{p}_{l 2}\right)<\epsilon$, where $\epsilon$ is the diameter of the cables. For the sake of simplicity, we consider cables of a negligible diameter, i.e., $\epsilon=0$. The roots of the function are then found using

$$
\begin{aligned}
& \underset{\lambda_{k, l}}{\operatorname{minimise}} \zeta\left(\mathbf{p}_{k 1}, \mathbf{p}_{k 2}, \mathbf{p}_{l 1}, \mathbf{p}_{l 2}\right) \\
& \text { subject to } 0 \leq \lambda_{k, l} \leq 1
\end{aligned}
$$

for the $\frac{m}{2}(m-1)$ possible pairs of cables, i.e., for $k, l \in\{1, \ldots, m\}, k<l$. If no root is found, then $\lambda_{k, l}=1$. Ultimately, when considering simultaneously wrench feasibility and the possibility of interference between any cable pair, the maximum point that can be reached on the trajectory is obtained as:

$$
\lambda=\mu \min \left\{\lambda_{s}, \lambda_{k, l}\right\}
$$

where $0 \leq \mu \leq 1$ is a factor introduced to account for discrepancies between the initial estimate of the robot geometry and its true geometry. It can be adjusted according to the level of trust in the initial approximation of the parameters, a high value representing confidence, and a low one, uncertainty. The resulting pose $X$ can finally be obtained using Eqs. (5a) and (5b). Since the optimisations (13) and (15) both starts from $X_{0}$, known to be located within the workspace of the robot, a feasible solution is always found, the worst case scenario being that $X_{j}=X_{0}$, i.e., $\lambda=0$.

Once the list of poses and their associated trajectories is generated, the focus can shift to the problem of tracking them with the robot. 


\section{Control}

In order to move the end effector to the computed calibration poses before the robot is fully calibrated, a control scheme capable of dealing with errors in the initial estimate of the geometric parameters is necessary. Thus, control is achieved by a simple low-level velocity controller, inspired by [2], driven by a Cartesian position controller. The block diagram of the control algorithm is presented in Fig. 2. To ensure valid cable measurements, this controller purpose is to keep the cables thaught, while trying to reach the desired pose.

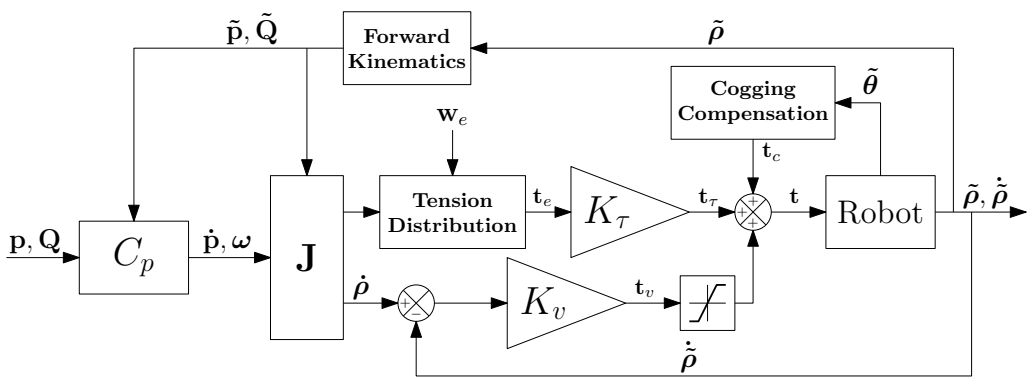

Fig. 2. Control scheme used to perform the calibration

The controller $C_{p}$ generates trajectories with trapezoidal speed profiles $\dot{\mathbf{p}}$ and $\boldsymbol{\omega}$ in order to reach the desired Cartesian position $\mathbf{p}$ and orientation $\mathbf{Q}$. The matrix $\mathbf{J}=-\mathbf{W}^{T}$ is then used to transform the desired Cartesian and angular speeds into articular speeds. A set of feasible cable tensions $\tau_{e}$ is generated from $\mathbf{w}_{e}$ using quadratic programming. The algorithm is concretely implemented using a $\mathrm{C}$ program generated with $C V X G E N$ [8]. The gain $K_{\tau}$ is derived from the geometry of the winches and used to link the desired cable tension to the motor torque, while $K_{v}$ is the gain of the low-level proportional speed controller. It should be noted than $K_{\tau}$ could be replaced by a closed loop tension controller. To address undesirable speed variation issues caused by the cogging ripple of the permanent magnets motors used in section 4, a cogging compensation block was introduced in the model. Even though this addition produced satisfying results, it will not be discussed in detail in this paper. The controlled motor torque is then given by the sum of the cogging compensation torque $\mathbf{t}_{c}$, the torque $\mathbf{t}_{\tau}$ needed to achieve the desired set of tensions and the torque $\mathbf{t}_{v}$ generated by the low-level proportional speed controller. A saturation is placed on $\mathbf{t}_{v}$ to prevent any excessive unwinding of the cables that could be caused by modeling errors, since the mechanism that will next be presented for validation is backdrivable.

\section{Experiment}

To test the performance of the proposed method, a validation is carried out on an existing CDPR. The setup consists of eight winches, each mounted on a 
motorised linear axis, allowing it to be easily reconfigured. The winches were designed to operate in industrial-like conditions and to withstand a large payload. They are driven by Parker MPP0923D motors coupled to 4:1 gearboxes. The size of the gantry is approximately $5 \mathrm{~m} \times 4 \mathrm{~m} \times 3 \mathrm{~m}$ and the winches are brought as close as physically possible to the corners of the structure.

The end effector is a $0.2 \mathrm{~m}, \times 0.2 \mathrm{~m} \times 0.6 \mathrm{~m}$ prism. The cables are made of $\varnothing 5 \mathrm{~mm}$ Dyneema SK75 synthetic rope. As can be seen in Fig. 3, they are attached to the end effector in a way that allows the control of its orientation. A self-contained vision module consisting of a 1.3 megapixel Basler ace acA1300$30 \mathrm{gm}$ camera, a battery and a Raspberry P $i$ computer is located inside the latter. The camera is oriented towards the markers, placed on the ground for practical reasons. For generality's sake, the markers are not considered to be located on a common plane. One marker is chosen as the reference for the whole map.

For the measurements, the ground truth is provided by a Faro X130 laser scanner with a ranging error of $\pm 2 \mathrm{~mm}$ (at $1 \sigma)$.

\subsection{Trials and results}

At first, the camera is calibrated using the Aruco calibration board, laminated on a flat plastic surface, resulting in a mean reprojection error is 0.51 pixels. Then, three different lists of random calibration poses (A, B and C) are generated using $\mu=0.8$ and a length of $n=100$ samples. The practical choice of $\mathcal{O}_{w}$ coincident with $\mathcal{O}_{m}$ is made. Data is then gathered in the form of relative cable length and pictures of the marker map are taken using the camera. Once the robot covered all calibration poses, the marker map geometry is established. Fig. 4 shows an example of the reconstructed camera poses and markers locations. Using the reconstructed camera poses, the calibration is performed by solving Eq.(3) using the trust-region-reflective algorithm provided by the lsqnonlin MATLAB function. The resulting geometric parameters are then updated, and the accuracy is tested for ten witness poses, shown in Fig. 5 .

A second calibration, for comparison purposes, is performed again but excluding the camera data, i.e., using only cable measurements. The second method, described in $[2,6]$, relies exclusively on the motor encoders of fully constrained
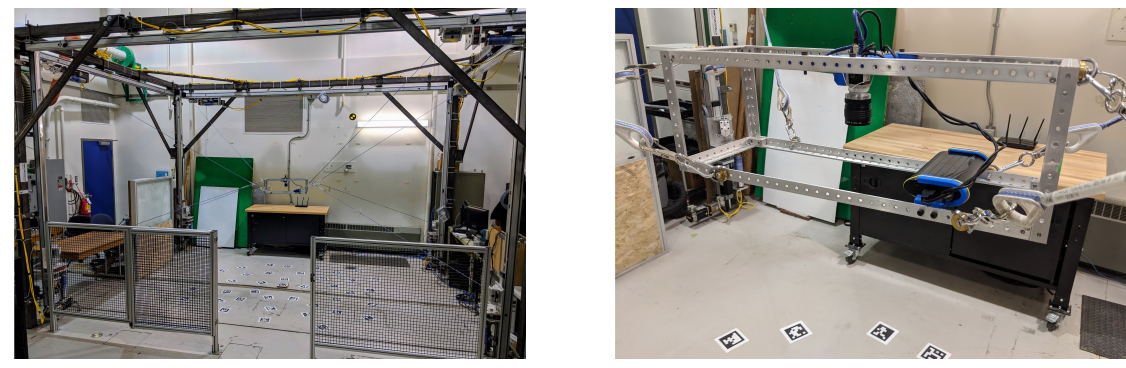

Fig. 3. Experimental setup: on the left, an overall view of the gantry and markers located on the ground. On the right, the end effector and the embedded vision system 


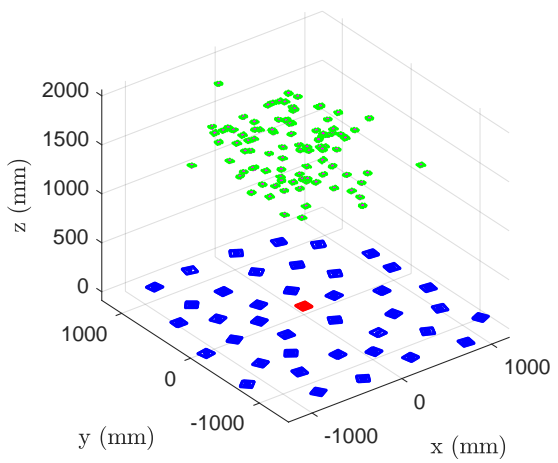

Fig. 4. Reconstructed marker map and camera poses

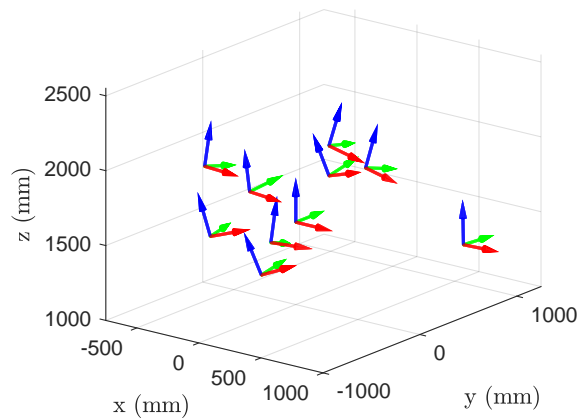

Fig. 5. Prescribed poses for calibration validation

CDPR architecture and as stated above, it can only be applied to robots with more cables than degrees of freedom. Without an external reference, the mechanism geometry is unconstrained. To resolve this indeterminacy and to ensure consistancy, six components of the $\mathbf{a}_{i}$ vectors are prescribed from the values obtained in the previous step. The end effector pose previously computed using the camera is also provided to the optimisation algorithm as an initial estimation of the solution to avoid convergence to a different local minimum. Upon completion of the calibration, the accuracy is tested for the same ten witness poses.

The error is determined by the difference between the forward kinematics problem (FK) result and an external measurement between the end effector and the reference marker, provided by the laser scanner. The optimisation convergence is expressed by $r$, which is defined as the fourth root of the residual of Eq. (3), divided by the number of calibration poses $n$.

Table 1 presents the compiled performance results. The pose is measured relative to the marker map (R.t. marker) used for calibration. To ensure proper comparison between the two calibration methods, and to prevent any bias caused by the camera from degrading the accuracy over the entire workspace, the pose 
is also measured relative to the first pose (R.t. $1^{\text {st }}$ pose). It should be noted that according to the ground truth measurements, errors in both position and orientation do not seem to be predominant in one particular direction.

Table 1. Calibration results

\begin{tabular}{|c|c|c|c|c|c|c|c|c|c|c|}
\hline \multirow[t]{3}{*}{ Data set } & & \multirow[t]{3}{*}{$\mathbf{r}(\mathbf{m m})$} & \multicolumn{4}{|c|}{ Position error ( $\mathrm{mm})$} & \multicolumn{4}{|c|}{ Orientation error (deg) } \\
\hline & & & \multicolumn{2}{|c|}{ R.t. marker } & \multicolumn{2}{|c|}{ R.t. $1^{\text {st }}$ pose } & \multicolumn{2}{|c|}{ R.t. marker } & \multicolumn{2}{|c|}{ R.t. $1^{\text {st }}$ pose } \\
\hline & & & Mean & Std & Mean & Std & Mean & Std & Mean & Std \\
\hline \multirow{3}{*}{$\begin{array}{c}\text { Proposed } \\
\text { method } \\
\text { (with camera) }\end{array}$} & $\mathrm{A}$ & 12.9 & 60.9 & 8.1 & 25.8 & 7.8 & 2.1 & 0.8 & 1.3 & 0.9 \\
\hline & $\mathrm{B}$ & 14.1 & 64.5 & 14.0 & 27.4 & 8.2 & 2.3 & 0.5 & 1.3 & 0.7 \\
\hline & $\mathrm{C}$ & 15.6 & 59.5 & 17.2 & 30.5 & 11.1 & 2.9 & 0.6 & 1.6 & 1.2 \\
\hline \multirow{3}{*}{$\begin{array}{c}\text { Cable lengths } \\
\text { only }[2,6]\end{array}$} & $\mathrm{A}$ & 3.8 & 278.3 & 22.0 & 28.6 & 11.2 & 4.6 & 1.8 & 2.9 & 2.0 \\
\hline & $\mathrm{B}$ & 4.2 & 268.2 & 24.0 & 28.7 & 16.0 & 3.2 & 1.2 & 2.7 & 1.0 \\
\hline & $\mathrm{C}$ & 4.0 & 984.5 & 25.6 & 110.1 & 32.1 & 7.6 & 4.4 & 5.7 & 1.1 \\
\hline
\end{tabular}

Additionally, for each of the 10 validation poses of the eye-on-hand calibrations, a snapshot of the marker map is taken using the camera. The resulting images can be compared to the previously established marker map geometry for the purpose of validating the camera pose reconstruction. Because the pose of the camera cannot be directly picked up, we measured instead the end effector pose. Table 2 summarises the errors obtained using the laser scanner data as a reference while Table 3 presents the discrepancy between the poses obtained with the camera measurements alone, and those obtained through the cable lengths, after calibration. In the following section, relationships between these data and the calibration results will be discussed.

Table 2. Camera pose errors, relative to ground-truth

\begin{tabular}{cccccc}
\hline Data set & \multicolumn{2}{c}{ Position error $(\mathbf{m m})$} & & \multicolumn{2}{c}{ Orientation error $(\mathbf{d e g})$} \\
\cline { 2 - 3 } \cline { 5 - 6 } & Mean & Std & & Mean & Std \\
\hline A & 59.2 & 9.1 & & 1.8 & 0.4 \\
B & 61.5 & 12.4 & & 1.9 & 0.4 \\
C & 30.8 & 9.6 & & 1.5 & 0.4 \\
\hline
\end{tabular}

\subsection{Discussion}

From the results of Table 1, it appears that the proposed eye-on-hand calibration method did improve the accuracy of the robot relative to the first pose, but this improvement is only statistically significant for some data sets. It can also be 
Table 3. Camera pose inconsistencies, relative to FK solution

\begin{tabular}{cccccc}
\hline Data set & \multicolumn{2}{c}{ Position error $(\mathbf{m m})$} & & Orientation error $(\mathbf{d e g})$ \\
\cline { 2 - 3 } \cline { 5 - 6 } & Mean & Std & & Mean & Std \\
\hline A & 8.4 & 4.2 & & 1.3 & 0.4 \\
B & 8.2 & 3.5 & & 1.2 & 0.5 \\
C & 32.2 & 10.3 & & 2.3 & 0.5 \\
\hline
\end{tabular}

seen by looking at the data set $\mathrm{C}$ that, when compared to the calibration method using only measurement redundancy, the use of the camera can prevent excessive divergence of the geometric parameters. Upon one-way analysis of variance using a significance level of 0.05 , the positioning accuracy was improved only for the dataset $\mathrm{C}$, while the orientation accuracy was improved for all datasets.

It can be observed from Table 3 that the FK solution is generally consistent with the reconstructed camera pose, especially in the case of the mean position error of data sets A and B. However, Table 2 shows that the same camera pose estimation is subject to errors in an order of magnitude similar to those measured after calibration, both in orientation and in translation. This suggests that the precision of the camera pose reconstruction is limiting the accuracy of the calibration. Improving the resolution and calibration of the vision system could therefore lead to improvements in calibration and thus in positioning accuracy.

\section{Conclusion}

This paper presents an investigation into the use of an eye-on-hand calibration method, for determining the geometric parameters of CDPRs. To this end, a list of reachable poses was generated based on an estimate of the CDPR geometry. A control scheme was adapted to allow the end effector to reach each of these poses. Finally, the method was experimentally validated on a medium sized fully constrained CDPR. While the worst relative mean position and orientation errors measured were respectively of $30.5 \mathrm{~mm}$ and $1.6^{\circ}$, the results suggest that the positioning accuracy of the calibrated CDPR could be increased by improving the accuracy of the reconstruction of the camera pose.

\section{Acknowledgments}

This work is financially supported by the Fonds de recherche du Québec - Nature et technologies (FRQNT), under the grant number 2016-PR-188869.

\section{References}

1. J. A. Dit Sandretto, D. Daney, and M. Gouttefarde. Calibration of a fullyconstrained parallel cable-driven robot. In 19th CISM-IFToMM Symposium on Robot Design, Dynamics, and Control (ROMANSY 2012), Paris, France, 2012. 
2. A. Fortin-Côté, P. Cardou, and C. Gosselin. An admittance control scheme for haptic interfaces based on cable-driven parallel mechanisms. In Proceedings - IEEE International Conference on Robotics and Automation, pages 819-825, 2014.

3. S. Garrido-Jurado, R. Muñoz-Salinas, F. J. Madrid-Cuevas, and R. MedinaCarnicer. Generation of fiducial marker dictionaries using Mixed Integer Linear Programming. Pattern Recognition, 51:481-491, 2016.

4. J.-B. Izard, M. Gouttefarde, M. Michelin, O. Tempier, and C. Baradat. A Reconfigurable Robot for Cable-Driven Parallel Robotic Research and Industrial Scenario Proofing. In T. Bruckmann and A. Pott, editors, Cable-Driven Parallel Robots, pages 135-148. Springer Berlin Heidelberg, Berlin, Heidelberg, 2013.

5. W. Khalil and S. Besnard. Self calibration of Stewart-Gough parallel robots without extra sensors. IEEE Transactions on Robotics and Automation, 15(6):11161121, 1999.

6. D. Lau. Initial Length and Pose Calibration for Cable-Driven Parallel Robots with Relative Length Feedback. In C. Gosselin, P. Cardou, T. Bruckmann, and A. Pott, editors, Cable-Driven Parallel Robots, pages 140-151, Cham, 2018. Springer International Publishing.

7. R. K. Lenz and R. Y. Tsai. Calibrating a Cartesian robot with eye-on-hand configuration independent of eye-to-hand relationship. IEEE Transactions on Pattern Analysis and Machine Intelligence, 11(9):916-928, sep 1989.

8. J. Mattingley and S. Boyd. CVXGEN: A code generator for embedded convex optimization. Optimization and Engineering, 13(1):1-27, 2012.

9. R. Meziane, P. Cardou, and M. J. Otis. Cable interference control in physical interaction for cable-driven parallel mechanisms. Mechanism and Machine Theory, 132:30-47, 2019.

10. P. Miermeister and A. Pott. Auto Calibration Method for Cable-Driven Parallel Robots Using Force Sensors. In J. Lenarcic and M. Husty, editors, Latest Advances in Robot Kinematics, pages 269-276. Springer Netherlands, Dordrecht, 2012.

11. R. Muñoz-Salinas, M. J. Marín-Jimenez, E. Yeguas-Bolivar, and R. MedinaCarnicer. Mapping and localization from planar markers. Pattern Recognition, 73:158-171, 2018.

12. A. J. Patel and K. F. Ehmann. Calibration of a hexapod machine tool using a redundant leg. International Journal of Machine Tools and Manufacture, 40(4):489512, mar 2000.

13. P. Renaud, N. Andreff, F. Marquet, and P. Martinet. Vision-based kinematic calibration of a $\mathrm{H} 4$ parallel mechanism. 2003 IEEE International Conference on Robotics and Automation (Cat. No.03CH37422), 1:1191-1196, 2003.

14. F. J. Romero-Ramirez, R. Muñoz-Salinas, and R. Medina-Carnicer. Speeded up detection of squared fiducial markers. Image and Vision Computing, 76:38-47, 2018.

15. H. Zhuang, O. Masory, and J. Yan. Kinematic calibration of a Stewart platform using pose measurements obtained by a single theodolite. Intelligent Robots and Systems 95. 'Human Robot Interaction and Cooperative Robots', Proceedings. 1995 IEEE/RSJ International Conference on, 2:329-334 vol.2, 1995.

16. H. Zhuang, J. Yan, and O. Masory. Calibration of Stewart platforms and other parallel manipulators by minimizing inverse kinematic residuals. Journal of Robotic Systems, 15:395-405, 1998. 